\title{
Advantages of PSWF-based models for UWB systems
}

\author{
Wouter Dullaert ${ }^{1}$ Hendrik Rogier ${ }^{2}$ Freek Boeykens ${ }^{3}$
}

\begin{abstract}
The frequency dependency of signals plays a very important role in Ultra Wideband (UWB) systems. Being able to accurately model this frequency dependency can provide a multitude of benefits, especially for UWB systems in medical applications. The model, based on prolate spheroidal wave functions (PSWFs), presented in this contribution improves greatly upon the model relying on discrete prolate spheroidal sequences that was previously presented by the authors: it offers a much more compact representation, while retaining the same robustness to noise. Furthermore, with the improved model, a cascade of signals can be evaluated directly using the model coefficients. All of these properties can be of use when analysing UWB systems.
\end{abstract}

\section{INTRODUCTION}

Ultra Wideband (UWB) communication systems play a very important role in the current movement to ubiquitous computing. Its low power requirements and high bandwidth makes it ideal for close range communications where battery life is of the utmost importance. Especially in medical applications it could enable the permanent monitoring of patients without the need for excessive wiring.

However, the advantages of UWB systems, being high bandwidth and low power consumption, provide various new challenges for the system design. Antenna design is tradiationally handled as a smallband problem: apart from the return loss, all the frequency dependency of all antenna parameters is ignored. In the case of UWB systems, this assumption is no longer valid, the frequency dependency of all parameters must be taken into account, which greatly increases the amount of data that needs to be handled during the design phase. Furthermore, the low spectral density of UWB systems means that great care needs to be taken to prevent noise from disturbing the data. Any form of representing UWB data should be able to handle, and preferably reduce, the noise contribution.

In this paper we would like to propose a model for the frequency depedency of UWB systems, based on prolate spheroidal wave functions (PSWfs). It is an improvement of the model presented by the authors in [1], which modeled frequency dependency using discrete prolate spheroidal sequences (DPSSs), the discrete counterpart of PSWFs. The model has a lot of properties which help to overcome the difficulties of UWB system design and analysis. In Section 2 we will briefly present the theory behind the model. In the following section, Section 3, the various properties and advantages of the model are illustrated. Finally, in Section 4 , we present our conclusions.

\section{PSWF-BASED MOdEL}

\subsection{Prolate Spheroidal Wave Functions}

The basic asumption of the model is that all UWB data have a very short time response $s(t)$, i.e they are timelimited, but at the same time have a frequency spectrum $S(f)$ that is concentrated in a given region $[-B / 2, B / 2]$. This means that the data can be efficiently respresented as a series of prolate spheroidal wave functions (PSWFs), as in [2],

$$
S(f)=\sum_{k=0}^{\infty} A_{k} \psi_{k, c}(f)
$$

where the coefficients $A_{k}$ are obtained as follows:

$$
A_{k}=\int_{-B / 2}^{B / 2} S(f) \psi_{k, c}(f) d f
$$

where $\psi_{k, c}(f)$ is the PSWF of order $k$ with a timebandwidth product $c=2 t_{0} B$.

The PSWFs were found to be the solution to the following maximisation problem over all timelimited functions $S(f)$ by Slepian et al. in [3]:

$$
\max \frac{\int_{-B / 2}^{B / 2}|S(f)|^{2} d f}{\int_{-\infty}^{\infty}|S(f)|^{2} d f}
$$

In other words, the zero-th order PSWF $\psi_{0, c}$ is the timelimited funtion with the most energy in the frequency interval $[-B / 2, B / 2]$. When (3) is evaluated

\footnotetext{
${ }^{1}$ Department of Information Technology, Ghent University, Sint-Pietersnieuwstraat 41 B-9000 Ghent, email: wouter.dullaert@intec.ugent.be

${ }^{2}$ Department of Information Technology, Ghent University, Sint-Pietersnieuwstraat 41 B-9000 Ghent, email: hendrik.rogier@intec.ugent.be

${ }^{3}$ Department of Information Technology, Ghent University, Sint-Pietersnieuwstraat 41 B-9000 Ghent, email: freek.boeykens@intec.ugent.be
} 
over all timelimited functions $S(f)$ orthogonal to $\psi_{0, c}$, we obtain the first order PSWF $\psi_{1, c}$ as a solution, etc. This, combined with the fact that the $\psi_{k}(f)$ are complete and orthogonal over both frequency ranges $[-B / 2, B / 2]$ and $[-\infty, \infty]$, makes them extremely well suited to model UWB data.

\subsection{Discrete Prolate Spheroidal Sequences}

The DPSSs are the discrete version of the PSWFs: instead of continuous functions, they are discrete series. Most of the properties of the PSWFs have a discrete equivalent for the DPSSs. The most important difference for our purpose is that DPSSs are defined on a sample set of a certain length: the first order DPSS of length 100 is not the same as the first order DPSS of length 200. This means that a signal can only be reconstructed on the same fixed sample points at which it has been modeled, severy limiting the applications of the model. The DPSSs, however, have the advantage that their construction is much more straightforward than that of the PSWFs.

\section{Model Properties}

In [1], DPSSs were used to model the frequency dependency of a radiation pattern. While this model performs well, the fact that DPSSs are discrete series instead of continuous functions, such as the data we are trying to model, make it a suboptimal solution. By replacing the DPSSs by PSWFs, we will obtain a model that offers a much more compact representation, as demonstrated in Section 3.1, is just as good, if not more, robust to noise, as shown in Section 3.2, and that opens up new possibilities such as cascading different system components, Section 3.3.

\subsection{Compactness}

To demonstrate the compactness of the model we rely on measured data from the log-periodic antenna presented in [4]. The radiation pattern was sampled from $400 \mathrm{MHz}$ to $20 \mathrm{GHz}$, resulting in a dataset of 801 samples. We will project this data onto DPSSs of length 801 on the one hand and PSWFs on the other hand. The coefficient series is truncated after $99.9 \%$ of the signal energy is present in the coefficients.

Projecting the signal onto DPSSs results in 291 coefficients, as shown in Figure 1. We have included some higher order coefficients, Figure 1 in black, to demonstrate that the energy of these higher orders is indeed very low. In Figure 2, the original signal and the signal reconstructed from the DPSSs series is shown. We can see that the reconstruction quite good, but not perfect.

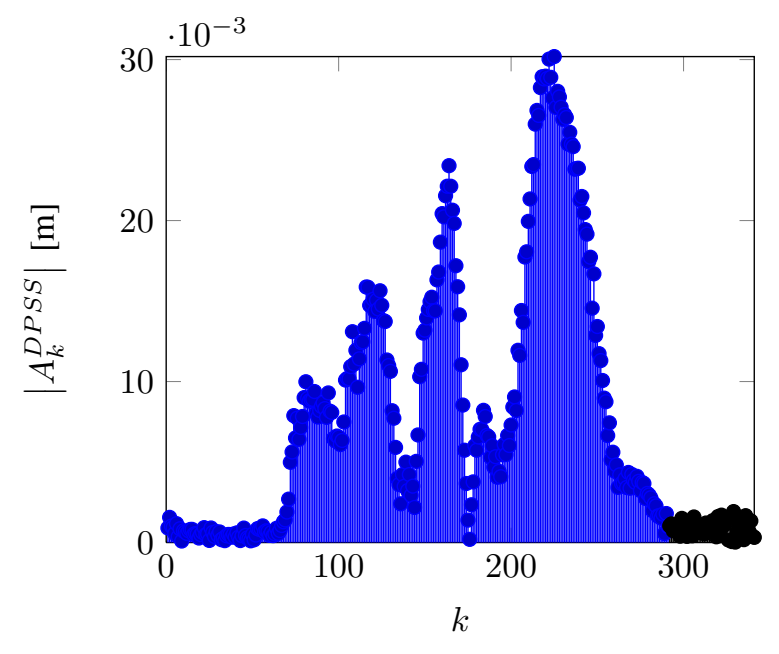

Figure 1. DPSS coefficients of the radiation pattern

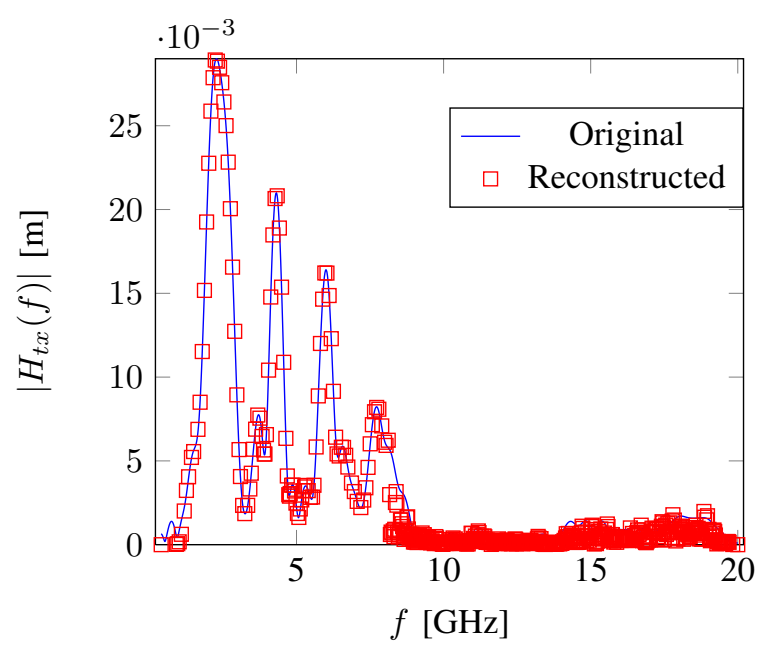

Figure 2. Original signal and DPSS reconstruction

Projecting the signal onto PSWFs results in 171 coefficients, as shown in Figure 3. Just like before we have included some higher order coefficients to demonstrate that the energy contained in the higher orders is indeed very low. In Figure 4, the original signal and the PSWF-reconstruction is shown. It can be easily verified that the reconstruction based on the PSWF coefficients is much more accurate, despite using only half the amount of modeling coefficients.

\subsection{Robustness To Noise}

In order to test the resistance of the model to noise, we have added white noise to the radiation pattern from Section 3.1. We obtained a filtered radiation pattern by projecting the noisy radiation pattern onto the DPSSs and PSWFs, respectively, and used the same amount of coefficients as before. In Figure 5 the noisy and DPSS filtered radiation pattern are shown. Figure 6 shows 


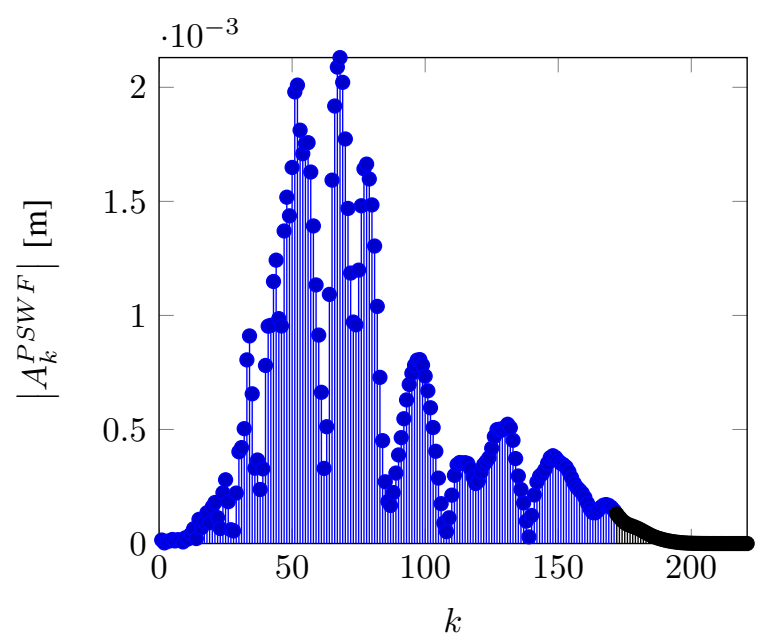

Figure 3. PSWF Coefficients of the radiation pattern

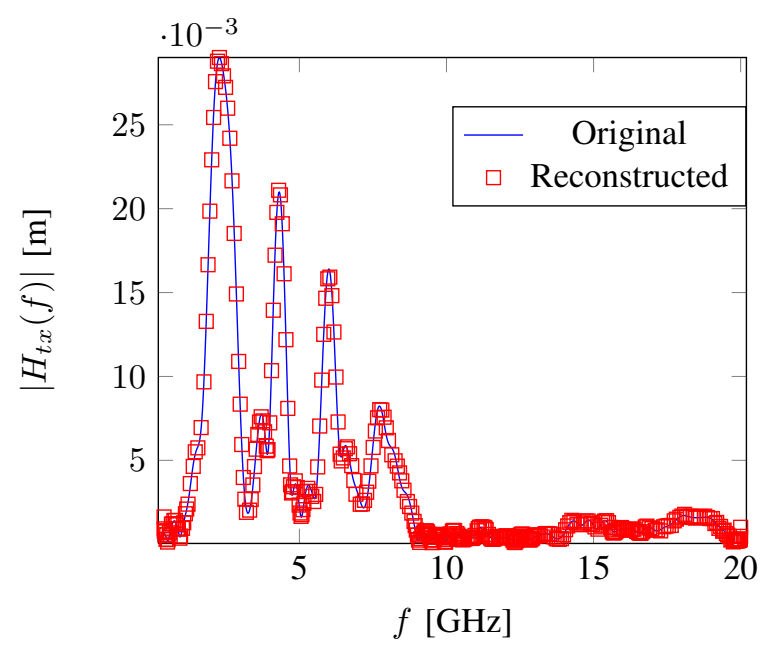

Figure 4. Original signal and PSWF reconstruction

the noisy and PSWF filtered radiation pattern. It can be seen that in both cases the filtered radiation pattern is in good agreement with the original radiation pattern and that the noise is considerably reduced. In the case of the PSWF filtered radiation pattern, there is very little noise left in the filtered data.

\subsection{Cascade}

As a final example, we will show that it is possible to compute the output of a cascade of systems using only their PSWF model coefficients. This is also possible with DPSSs, but because of the fact that DPSSs are dependent on their length, all parts of the cascade would need to be sampled at exactly the same points in the time and frequency domain, thereby greatly reducing the amount of potential applications.

Let us consider the result $P_{\text {out }}(f)$ of a cascade of a

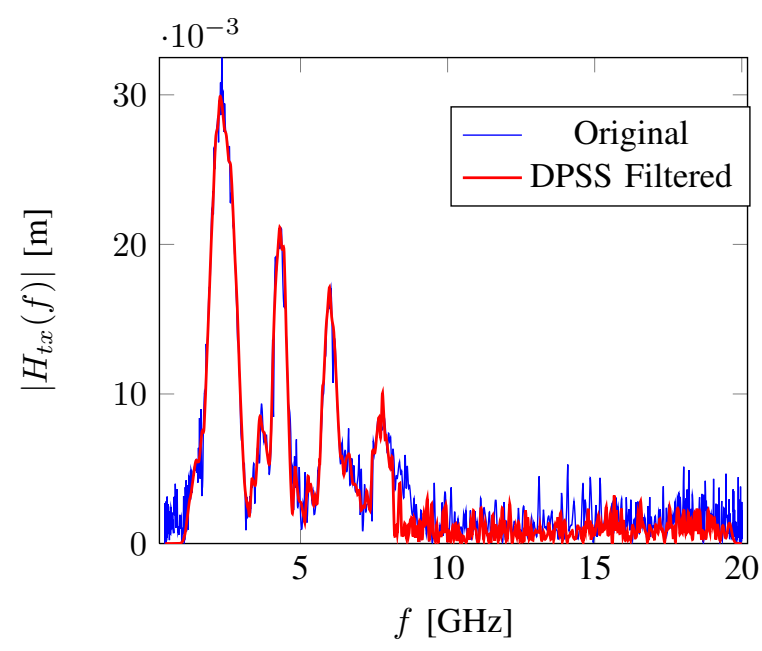

Figure 5. Noisy and DPSS filtered radiation pattern

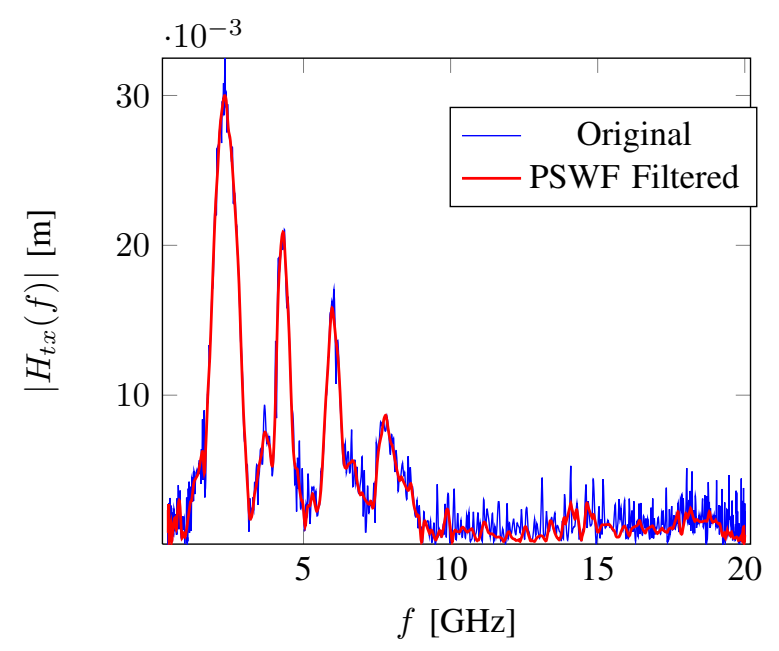

Figure 6. Noisy and PSWF filtered radiation pattern

pulse $P_{i n}(f)$ with a radiation pattern $H_{t x}(f)$ :

$$
\begin{aligned}
P_{\text {out }}(f) & =H_{t x}(f) P_{\text {in }}(f) \\
& =\sum_{k=0}^{K} A_{k} \psi_{k}(f) \sum_{l=0}^{L} B_{k} \psi_{l}(f) \\
& =\sum_{k, l=0}^{K, L} A_{k} B_{l} \psi_{k}(f) \psi_{l}(f) \\
& =\sum_{m} C_{m} \psi_{m}(f)
\end{aligned}
$$

where $A_{k}$ are the PSWF coefficients of $H_{t x}(f)$ and $B_{k}$ are the PSWF coefficients of $P_{i n}(f)$. Combining (2) and (7) we get:

$$
C_{m}=\int_{-B / 2}^{B / 2} \sum_{k, l=0}^{K, L} A_{k} B_{l} \psi_{k}(f) \psi_{l}(f) \psi_{m}(f) d f
$$




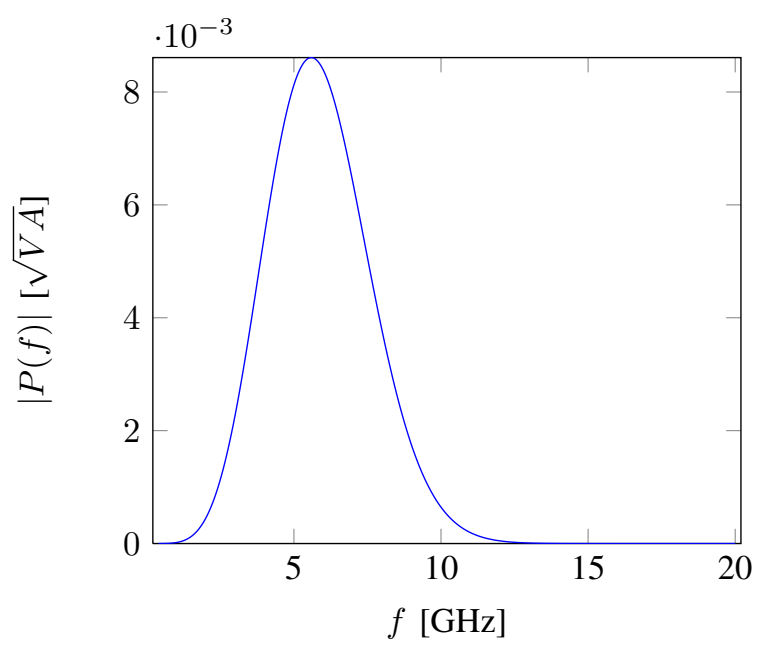

Figure 7. Spectrum of a UWB compliant pulse

$$
\begin{aligned}
& =\sum_{k, l=0}^{K, L} A_{k} B_{l} \int_{-B / 2}^{B / 2} \psi_{k}(f) \psi_{l}(f) \psi_{m}(f) d f(9) \\
& =\sum_{k, l=0}^{K, L} A_{k} B_{l} Q_{k, l, m}
\end{aligned}
$$

where $Q_{k, l, m}=\int_{-B / 2}^{B / 2} \psi_{k}(f) \psi_{l}(f) \psi_{m}(f)$ is a matrix containing the cross products of the PSWFs. As this matrix is independent from the input data and highly symetrical, it can easily be precomputed. (10) allows us to calculate the coefficients of the output pulse using only the coefficients of the input pulse.

As an example we will evaluate the cascade of the radiation pattern of the previous sections with the spectrum of a UWB compliant pulse $P_{i n}(f)$, [5]:

$$
P_{\text {in }}(f)=A_{c} f^{5} \exp -\frac{f^{2} \sigma^{2}}{2} j
$$

where $A_{c}$ is used to scale the pulse so that it fits the UWB spectral mask and $\sigma=51 \times 10^{-12}$. In Figure 7 the spectrum of the input pulse is shown. Figure 8 shows the output pulse $P_{\text {out }}(f)$, both computed conventionally and using the model. Both curves are in very good agreement. In fact, the reconstruction error is of the same magnitude as the largest reconstruction error of the input data, meaning that the cascading formula has a neglegible contribution to the overall error.

\section{Conclusion}

In this paper an improved model for the frequency dependency of UWB systems was presented. By projecting the data onto a weighted series of prolate

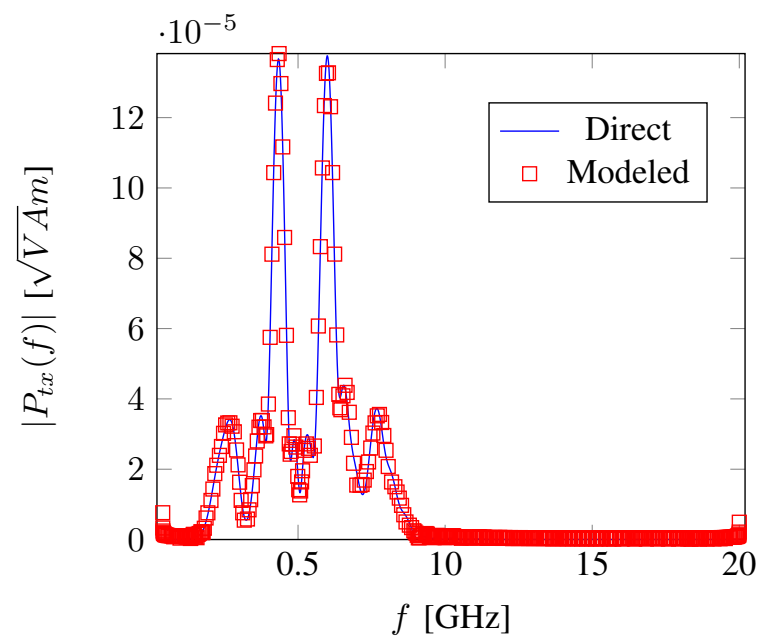

Figure 8. Spectrum of the output pulse

spheroidal wave functions (PSWFs), we obtain a compact representation that is very robust to noise. It was shown that the model performs much better than the projection on discrete prolate spheroidal sequences (DPSSs), presented in [1]. We also showed that it is possible to evaluate the cascade of multiple UWB system components, using only their PSWF coefficients. This greatly expands the amount of applications for this model.

\section{References}

[1] W. Dullaert and H. Rogier, "Novel Compact Model for the Radiation Pattern of UWB Antennas Using Vector Spherical and Slepian Decomposition," IEEE Transactions on Antennas and Propagation, vol. 58, no. 2, pp. 287-299, 2010. [Online]. Available: http://ieeexplore.ieee.org/lpdocs/epic03/wrapper.htm? arnumber $=5345757$

[2] W. Dullaert, L. Reichardt, and H. Rogier, "Improved Detection Scheme for Chipless RFIDs Using Prolate Spheroidal Wave Function-based Noise Filtering," IEEE Antennas and Wireless Propagation Letters, vol. 10, no. c, pp. 472-475, 2011. [Online]. Available: http://ieeexplore.ieee.org/lpdocs/epic03/wrapper.htm? arnumber $=5767542$

[3] D. Slepian and H.O. Pollak, "Prolate Spheroidal Wave Functions, Fourier Analysis, and Uncertainty - I," Bell Systems Technology Journal, vol. 40, no. 1, pp. 43-64, Jan. 1961.

[4] W. Wiesbeck, G. Adamiuk, and C. Sturm, "Basic Properties and Design Principles of UWB Antennas," Proceedings of the IEEE, vol. 97, no. 2, pp. 372-385, 2009. [Online]. Available: http://ieeexplore.ieee.org/lpdocs/epic03/wrapper.htm? arnumber $=4802200$

[5] H. Sheng, P. Orlik, A. M. Haimovich, J. Cimini L.J., and J. Zhang, "On the spectral and power requirements for ultrawideband transmission," in Proc. IEEE International Conference on Communications ICC '03, vol. 1, 2003, pp. 738-742. 